\title{
Meningococcal disease in NSW, \\ 1991-2011: trends in relation to meningococcal C vaccination
}

\author{
Erin Passmore $^{\mathrm{A}, \mathrm{B}, \mathrm{E}}$, Mark J. Ferson ${ }^{\mathrm{B}, \mathrm{C}}$ \\ and Sean Tobin ${ }^{\mathrm{D}}$ \\ ${ }^{\mathrm{A}}$ NSW Public Health Officer Training Program, NSW Ministry \\ of Health \\ ${ }^{\mathrm{B}}$ School of Public Health and Community Medicine, The University \\ of New South Wales \\ ${ }^{\mathrm{C}}$ Public Health Unit, South Eastern Sydney Local Health District \\ ${ }^{\mathrm{D}}$ Health Protection NSW \\ ${ }^{\mathrm{E} C o r r e s p o n d i n g ~ a u t h o r . E m a i l: ~ e p a s s @ d o h . h e a l t h . n s w . g o v . a u ~}$
}

Abstract: Aim: To review the epidemiology of invasive meningococcal disease in NSW for the period 1991-2011, in particular since the introduction of the meningococcal $\mathrm{C}$ vaccination program in 2003. Methods: We undertook a descriptive analysis of NSW notifications of invasive meningococcal disease for the period 2003-2011, and explored long-term changes in the epidemiology of invasive meningococcal disease over the period 1991-2011. Results: In the period 2003-2011, there were 1009 notifications of invasive meningococcal disease in NSW, an average annual rate of 1.6 per 100000 population. Notification rates were highest in the $0-4$ and 15-19-year age groups (8.5 and 3.6 per 100000 population respectively). In the period 1991-2011, invasive meningococcal disease notifications increased between 1991 and 2000, peaking at 3.8 notifications per 100000 population in 2000 . Notifications have decreased since that time to 1.0 per 100000 population in 2011 , most markedly for serogroup $\mathrm{C}$ disease since the introduction of the meningococcal $\mathrm{C}$ vaccination program in 2003. Meningococcal C notifications reduced from 54 in 2002 ( 0.8 per 100000 population) to two in 2011 ( 0.03 per 100000 population). Meningococcal $\mathrm{C}$ deaths have also decreased, from nine in 2002 to zero in 2011. The greatest reduction in meningococcal $\mathrm{C}$ notifications has been in those aged 1-19 years, the target group for the vaccination program. Meningococcal B notifications have also decreased over the study period, however serogroup B remains the predominant serogroup for invasive meningococcal disease in NSW. Conclusion: Notification rates of invasive meningococcal disease have decreased in NSW since 2000. Rates of serogroup C disease have decreased since the introduction of the meningococcal $C$ vaccination program in 2003. Most of the burden of invasive meningococcal disease in NSW is now due to serogroup B disease.

Invasive meningococcal disease (IMD) is caused by the bacteria Neisseria meningitidis. There are several serogroups of $N$. meningitidis. Serogroup B disease is the most common in Australia; other less common serogroups are A, C, W135 and Y. Disease due to serogroups A, C, $\mathrm{W} 135$ and $\mathrm{Y}$ is vaccine-preventable. There is no licensed vaccine for serogroup $B$ disease.

The meningococcal $\mathrm{C}$ conjugate vaccine protects against serogroup C disease. In 2003, the Australian Government commenced a national meningococcal $\mathrm{C}$ vaccination program with the aim of vaccinating all people aged 1-19 years. Since 2003, the meningococcal C conjugate vaccine has been included in free routine vaccination for all children at 12 months of age. Until June 2006, this was supplemented by a vaccination 'catch-up' program through general practice and school-based vaccination programs for those born between 1984 and 2001. ${ }^{1}$

Uptake of the meningococcal C conjugate vaccine through routine infant vaccination is high. Coverage for 24-month olds has remained at over $90 \%$ since first calculated for NSW in $2006 .{ }^{2}$ Data on the uptake of the general practitionerbased catch-up program is not available. A NSW Health evaluation of the school-based catch-up program in 20032004 indicated that $76 \%$ of high school students and $76 \%$ of primary school students were vaccinated (unpublished data).

In addition to the serogroup $\mathrm{C}$ conjugate vaccine, quadrivalent meningococcal conjugate and polysaccharide vaccines against serogroups A, C, W135 and Y are available 
but are not recommended for routine use. A single dose of a quadrivalent meningococcal conjugate vaccine is recommended for people in certain situations, such as travellers who intend visiting areas where epidemics of serogroup A, W135 or Y disease are frequent (e.g. sub-Saharan Africa), for pilgrims to the Hajj in Saudi Arabia, and for laboratory workers who frequently handle $N$. meningitidis. The vaccines are also recommended for close contacts of cases with disease caused by serogroup A, W135 or Y and for people with high-risk medical conditions. ${ }^{3}$ Quadrivalent meningococcal conjugate vaccines are preferred to polysaccharide meningococcal vaccines due to their greater duration of induced immunity. ${ }^{4}$

The epidemiology of IMD notifications in NSW for the period 1991-2002 has been reported previously., ${ }^{5,6}$ To explore changes in IMD epidemiology, particularly in relation to the introduction of the serogroup $\mathrm{C}$ vaccination program in 2003, this report presents an overview of the epidemiology of IMD in NSW for the period 1991-2011, and presents new data for the period 2003-2011.

\section{Methods}

In NSW, a case of IMD is defined according to national guidelines (Box 1). Hospitals and laboratories are required to notify cases of IMD to Public Health Units under the NSW Public Health Act 2010 (and previously under the NSW Public Health Act 1991). Public Health Units investigate all notified cases of IMD to collect risk information and implement control measures to prevent secondary cases of disease. Confirmed and probable cases of IMD are entered into the NSW Notifiable Conditions Information Management System (NCIMS), a statewide database of notifiable conditions that is maintained by NSW Health. Notifications of confirmed and probable cases of IMD with onset between 1 January 1991 and 31 December 2011 are presented in this report. Annual notification rates were calculated using Australian Bureau of Statistics mid-year estimated resident populations, obtained through the NSW Health Outcomes Information and Statistical Toolkit (HOIST). Cases were analysed by year of onset, gender, age group, disease syndrome (meningitis and/or septicaemia, based on presence of $N$. meningitidis in diagnostic specimens), serogroup and disease outcome.

Analyses were performed using SAS (version 9.2, SAS Institute, Cary, NC, USA) and Microsoft Excel 2007.

\section{Results}

Trends in IMD notifications, 1991-2011

There was a sustained increase in annual notification rates in the period 1991-2000, followed by a sustained decrease in the period 2000-2011. The notification rate decreased by $74 \%$ from a peak of 3.8 per 100000 population in 2000
Box 1. Surveillance case definition for invasive meningococcal disease, Australia

\section{CONFIRMED CASE}

A confirmed case requires either:

1. Laboratory definitive evidence

OR

2. Laboratory suggestive evidence AND clinical evidence

Laboratory definitive evidence

1. Isolation of Neisseria meningitidis from a normally sterile site

OR

2. Detection of specific meningococcal DNA sequences in a specimen from a normally sterile site by nucleic acid amplification testing

Laboratory suggestive evidence

1. Detection of Gram-negative diplococci in Gram stain of specimen from a normally sterile site or from a suspicious skin lesion

OR

2. High titre $\lg M$ or significant rise in $\lg M$ or $\lg G$ titres to outer membrane protein antigens of Neisseria meningitidis

Clinical evidence (for a confirmed case)

Disease which in the opinion of the treating clinician is compatible with invasive meningococcal disease.

\section{PROBABLE CASE}

A probable case requires clinical evidence only.

Clinical evidence (for a probable case)

A probable case requires:

1. The absence of evidence for other causes of clinical symptoms

AND EITHER

2. Clinically compatible disease including haemorrhagic rash

OR

3. Clinically compatible disease AND close contact with a confirmed case within the previous 60 days

The national case definition was revised and endorsed by Communicable Diseases Network Australia in October 2007. Source: Communicable Diseases Network Australia. Meningococcal disease case definition, implemented July 2010. Available at: http://www.health.gov.au/internet/main/publishing. nsf/content/cda-surveil-nndss-casedefs-cd_mening.htm 
Table 1. Notifications of invasive meningococcal disease by year, annual rate per 100000 population, and case fatality rate, NSW, 1991-2011

\begin{tabular}{|c|c|c|c|c|}
\hline Year & $\begin{array}{c}\text { Notified } \\
\text { cases }\end{array}$ & $\begin{array}{l}\text { Annual } \\
\text { rate }\end{array}$ & $\begin{array}{c}\text { Notified } \\
\text { deaths }\end{array}$ & $\begin{array}{c}\text { Case fatality } \\
\text { rate }(\%)\end{array}$ \\
\hline 1991 & 128 & 2.2 & 3 & 2.3 \\
\hline 1992 & 121 & 2.0 & 8 & 6.6 \\
\hline 1993 & 153 & 2.5 & 11 & 7.2 \\
\hline 1994 & 142 & 2.3 & 15 & 10.6 \\
\hline 1995 & 113 & 1.8 & 7 & 6.2 \\
\hline 1996 & 161 & 2.6 & 7 & 4.3 \\
\hline 1997 & 218 & 3.5 & 7 & 3.2 \\
\hline 1998 & 184 & 2.9 & 17 & 9.2 \\
\hline 1999 & 217 & 3.4 & 14 & 6.5 \\
\hline 2000 & 249 & 3.8 & 14 & 5.6 \\
\hline 2001 & 232 & 3.5 & 7 & 3.0 \\
\hline 2002 & 213 & 3.2 & 17 & 8.0 \\
\hline 2003 & 198 & 3.0 & 13 & 6.6 \\
\hline 2004 & 146 & 2.2 & 5 & 3.4 \\
\hline 2005 & 137 & 2.0 & 9 & 6.6 \\
\hline 2006 & 102 & 1.5 & 6 & 5.9 \\
\hline 2007 & 109 & 1.6 & 5 & 4.6 \\
\hline 2008 & 80 & 1.1 & 3 & 3.8 \\
\hline 2009 & 92 & 1.3 & 4 & 4.3 \\
\hline 2010 & 74 & 1.0 & 5 & 6.8 \\
\hline 2011 & 71 & 1.0 & 4 & 5.6 \\
\hline Total & 3140 & 2.3 & 181 & 5.8 \\
\hline
\end{tabular}

down to 1.0 per 100000 population in 2011 (Table 1). The decrease in notifications occurred for both serogroup B and $\mathrm{C}$ disease (Figure 1). Serogroup B notifications peaked in 2002 at 1.6 per 100000 population; serogroup C notifications peaked in 2000 at 1.0 per 100000 population. Notifications of serogroups W135 and Y remained low and stable over this period, with an average of three serogroup W135 notifications and three serogroup Y notifications per year. The proportion of cases for which serogroup information is recorded has steadily increased over time, from $0 \%$ in 1991 to $74.6 \%$ in 2011, and the apparent increase in notifications of serogroup B and C disease from 1991 to 2000 partly reflects the increasing availability of serogroup information.

\section{Notifications of IMD, 2003-2011}

In the period 2003-2011, there were 1009 notifications of IMD in NSW, an average annual rate of 1.6 notifications per 100000 population. A total of $879(87.1 \%)$ were confirmed cases, while 130 (12.9\%) were probable cases. This average notification rate is substantially lower than in the previous study period 1991-2002 (2.8 per 100000 population). Characteristics of notified cases in the period 2003-2011 are shown in Table 2.

The highest rates of IMD occurred among children aged less than 5 years of age, who accounted for $33.5 \%$ of notifications (8.5 per 100000 population). Within this group, children aged under 12 months were at highest risk (17.9 notifications per 100000 population). A second smaller peak in the notification rate was seen in the

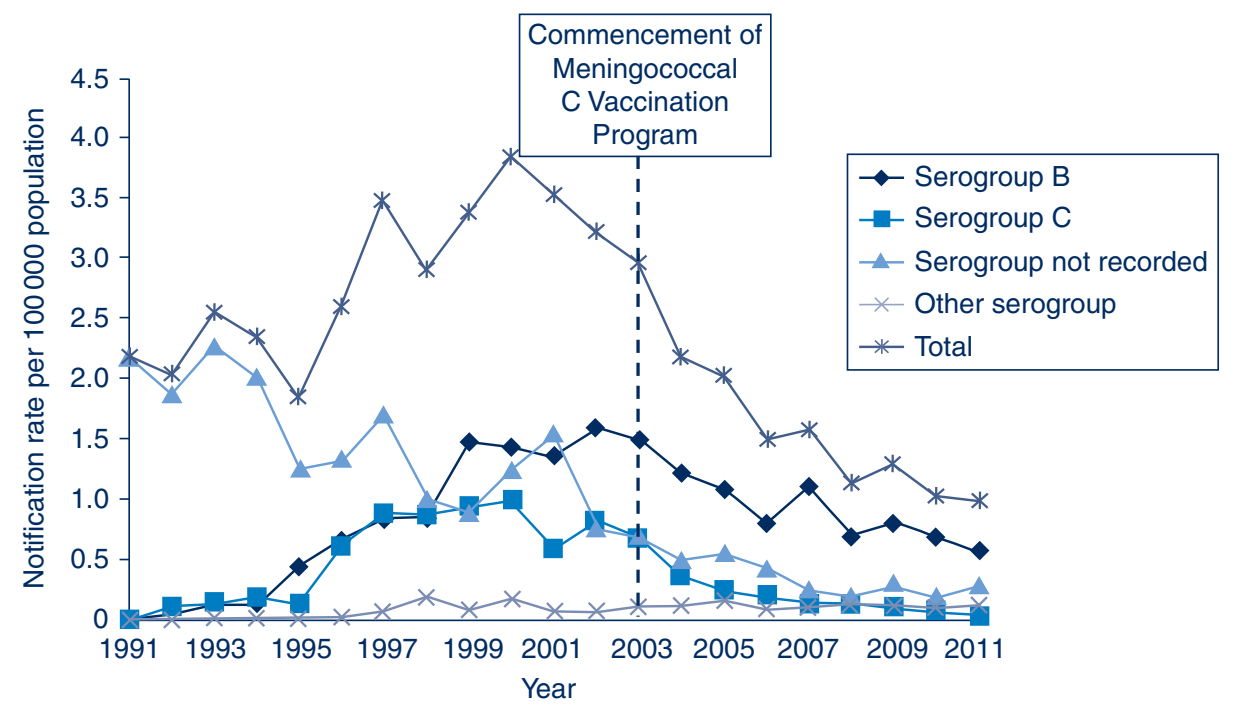

Figure 1. Notifications of invasive meningococcal disease by serogroup and year of onset, NSW, 1991-2011

Source: Notifiable Conditions Information Management System, NSW Health. 
Table 2. Characteristics of cases of invasive meningococcal disease notified in NSW, 2003-2011

\begin{tabular}{|c|c|c|c|c|c|}
\hline Case characteristics & Cases & $\begin{array}{c}\% \\
\text { total }\end{array}$ & $\begin{array}{l}\text { Average annual } \\
\text { rate per } 100000\end{array}$ & $\begin{array}{l}\text { Notified } \\
\text { deaths }\end{array}$ & $\begin{array}{c}\text { Case fatality } \\
\text { rate }(\%)\end{array}$ \\
\hline \multicolumn{6}{|l|}{ Gender } \\
\hline Female & 491 & 48.7 & 1.6 & 26 & 5.3 \\
\hline Male & 518 & 51.3 & 1.7 & 28 & 5.4 \\
\hline \multicolumn{6}{|l|}{ Age group (years) } \\
\hline$<1$ & 142 & 14.1 & 17.9 & 13 & 9.2 \\
\hline 1 & 82 & 8.1 & 10.4 & 0 & 0.0 \\
\hline $2-4$ & 114 & 11.3 & 4.8 & 2 & 1.8 \\
\hline $5-9$ & 68 & 6.7 & 1.7 & 1 & 1.5 \\
\hline $10-14$ & 47 & 4.7 & 1.1 & 1 & 2.1 \\
\hline $15-19$ & 150 & 14.9 & 3.6 & 5 & 3.3 \\
\hline $20-24$ & 98 & 9.7 & 2.3 & 3 & 3.1 \\
\hline $25-44$ & 129 & 12.8 & 0.7 & 8 & 6.2 \\
\hline $45-64$ & 115 & 11.4 & 0.8 & 12 & 10.4 \\
\hline $65-74$ & 31 & 3.1 & 0.7 & 5 & 16.1 \\
\hline $75-84$ & 25 & 2.5 & 0.8 & 3 & 12.0 \\
\hline $85+$ & 8 & 0.8 & 0.8 & 1 & 12.5 \\
\hline \multicolumn{6}{|l|}{ Syndrome } \\
\hline Meningitis & 276 & 27.4 & 0.45 & 4 & 1.4 \\
\hline Septicaemia & 464 & 46.0 & 0.75 & 40 & 8.6 \\
\hline Both meningitis and septicaemia & 169 & 16.7 & 0.27 & 9 & 0.9 \\
\hline Other/Unknown & 100 & 9.9 & 0.16 & 1 & 1.0 \\
\hline \multicolumn{6}{|l|}{ Serogroup } \\
\hline B & 580 & 57.5 & 0.9 & 28 & 4.8 \\
\hline $\mathrm{C}$ & 130 & 12.9 & 0.2 & 13 & 10.0 \\
\hline W135 & 40 & 4.0 & 0.1 & 5 & 12.5 \\
\hline $\mathrm{Y}$ & 31 & 3.1 & 0.0 & 2 & 6.5 \\
\hline Unknown, untypable & 228 & 22.6 & 0.4 & 6 & 2.6 \\
\hline Total & 1009 & & 1.6 & 54 & 5.4 \\
\hline
\end{tabular}

15-19 and 20-24-year age groups (3.6 and 2.3 notifications per 100000 population, respectively). The age distribution of cases is similar to the previous study period.

\section{Deaths}

In the period 1991-2011, whether the person with IMD died was recorded for $55 \%$ of notifications; cases with missing information regarding death were assumed not to have died. There were 181 notified deaths due to IMD in the period, which represents $5.8 \%$ of cases. Since 1991, annual case fatality rates have fluctuated between $2.3 \%$ and $10.6 \%$ (Table 1). In the period 2003-2011, there were 54 notified deaths due to IMD, which represents $5.4 \%$ of notifications for this period (Table 2). The proportion of cases who died was significantly higher for serogroup $\mathrm{C}$ compared to serogroup B disease (RR 2.1, 95\% CI 1.13.9), for cases presenting with septicaemia compared to meningitis (RR 5.9, 95\% CI 2.2-16.4), and for cases aged 45 years and over compared to younger cases (RR 3.0, 95\%
CI 1.7-5.0). There was no significant association between death and gender. These patterns are similar to those observed in the previous study period.

\section{Serogroup C disease}

Notifications of serogroup $\mathrm{C}$ disease have decreased dramatically since the introduction of the meningococcal $\mathrm{C}$ vaccination program in 2003 , from 54 in $2002(0.8$ per 100000 population) to two in 2011 ( 0.03 per 100000 population). Notifications of deaths due to serogroup $\mathrm{C}$ disease have also decreased, from nine in 2002 (the year before the vaccination program started) to zero in 2011. The proportion of serotyped cases of IMD that are serogroup C also decreased, from $33.1 \%$ in 2002 to $3.9 \%$ in 2011. In the same period, the proportion of serotyped cases that were serogroup B increased from $64.4 \%$ to $80.4 \%$, and other serogroups from $2.5 \%$ to $15.7 \%$.

The decline in serogroup $\mathrm{C}$ disease has occurred across all age groups. Immediately after the introduction of the 
meningococcal $\mathrm{C}$ vaccination program, notifications of serogroup C disease for 1-19-year olds (the target age group of the vaccination program) dropped dramatically, from 30 cases in 2002 to four cases in 2004, with less than five notifications per year since that time. There has also been a gradual decline in notifications in age groups not targeted in the vaccination program, from one case in 2002 to no cases in 2011 in children less than 12 months of age, and from 23 cases in 2002 to two cases in 2011 in those aged 20 years and over. Similarly, the proportion of serotyped cases that are serogroup $\mathrm{C}$ has decreased over time across all age groups. This decrease is most marked for 1-19-year olds, but is apparent even in unvaccinated children less than 12 months of age (Figure 2). Across the entire study period, the proportion of serotyped notifications that are serogroup $\mathrm{C}$ was lower in children under 12 months of age than for the older age groups.

Since the commencement of the meningococcal C vaccination program in 2003, meningococcal C vaccination status has been recorded for 89 (68.5\%) serogroup C notifications. There have been two notifications of serogroup C disease in vaccinated individuals, a 2-year old child in 2008, and a 9-year old child in 2009.

\section{Discussion}

Notifications of cases of IMD have become rare in NSW. Children aged under 5 years continue to be at highest risk of IMD, in particular children aged under 12 months, with young adults aged 15-24 years also at elevated risk. IMD notifications in NSW increased between 1991 and 2000, and have decreased since this time.

The greatest reduction in notifications and deaths has been for serogroup $\mathrm{C}$ disease since the introduction of the meningococcal $\mathrm{C}$ vaccination program in 2003. The marked decrease in serogroup $C$ disease in 1-19-year olds in 2003 and 2004 most likely reflects the impact of the vaccination program in the target age group. There was also a reduction in serogroup $\mathrm{C}$ notifications in other age groups not targeted in the vaccination program. This partly reflects the movement of vaccinated young adults into the older age group, but may also suggest protection through herd immunity as a consequence of the vaccination program. There have been only two notifications of serogroup $\mathrm{C}$ disease in vaccinated individuals, suggesting vaccine failure is uncommon. It is important to continue to collect information about the vaccination status of cases in order to monitor the effectiveness of the vaccine and appropriateness of the vaccination policy.

The epidemiology of IMD at a national level has been described elsewhere. ${ }^{7-9}$ Nationally, notification rates for IMD have decreased from 3.1 per 100000 population in 2002 to 1.1 per 100000 population in $2011 .{ }^{10}$ The dramatic reduction in rates of notifications of serogroup $\mathrm{C}$
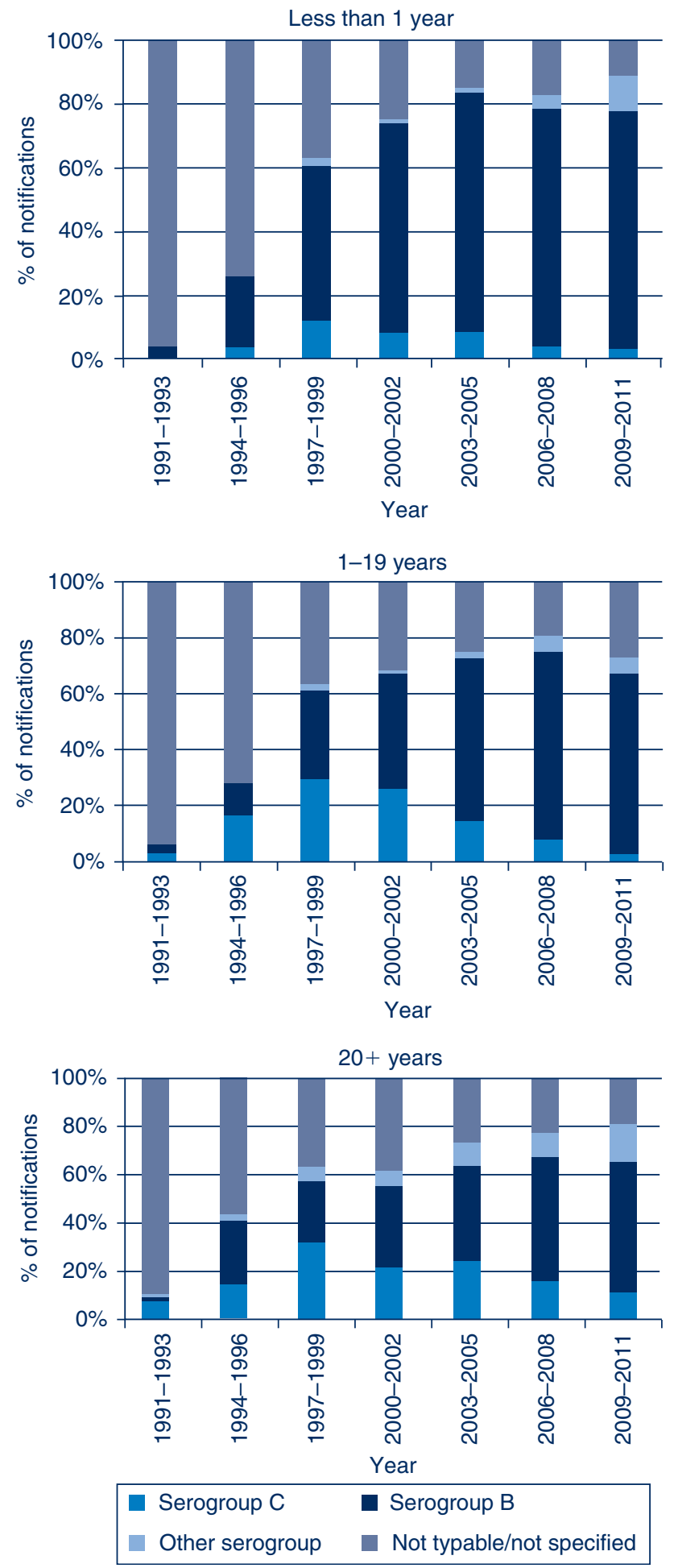

Figure 2. Notifications of invasive meningococcal disease by age, serogroup and year of onset, NSW, 1991-2011 Source: Notifiable Conditions Information Management System, NSW Health.

disease in NSW between 2002 and 2011 is comparable to rates observed nationally ${ }^{8}$ and in other countries where meningococcal $\mathrm{C}$ vaccination programs have been introduced. ${ }^{11-14} \mathrm{NSW}$, Australian and international data consistently indicate there has been no evidence of serogroup replacement following mass meningococcal C immunisation programs. ${ }^{15}$ 
Serogroup B remains the predominant serogroup for IMD in NSW and now accounts for $80 \%$ of cases. Research is underway to develop a vaccine for serogroup B disease, ${ }^{16}$ which would substantially reduce the burden of IMD in Australia and worldwide. Although the proportion of annual IMD notifications due to serogroup B disease has increased over time, the actual number of serogroup B notifications has declined since 2000 . This decrease is not likely to be related to the meningococcal $\mathrm{C}$ vaccination program but may reflect a natural drift in the virulence of meningococcal B strains in the community. ${ }^{6}$ Notifications for serogroups $\mathrm{Y}$ and $\mathrm{W} 135$ have remained low and stable.

Although notifications for IMD have reduced greatly, reducing deaths and disability remain key challenges in the management of IMD. IMD remains one of the leading infectious causes of death in children in Australia. Moreover, overseas studies indicate that $9 \%$ of children who survive IMD are left with major physical, cognitive and/or psychological disabilities, ${ }^{17}$ and $50 \%$ of survivors of adolescent IMD experience major long-term sequelae including skin scarring, mobility difficulty, and speech and hearing problems. ${ }^{18}$ Structured review of the management of individual IMD cases has been used to identify areas for improvement in the medical, laboratory and public health management of IMD cases ${ }^{19}$ and has the potential to improve outcomes for people with IMD.

\section{Conclusion}

This study used routinely collected surveillance data to describe the epidemiology of IMD in NSW, and to examine trends in relation to the introduction of the meningococcal $\mathrm{C}$ vaccination program. The incidence of meningococcal $\mathrm{C}$ has decreased substantially since the introduction of the meningococcal $\mathrm{C}$ vaccination program. A key challenge for reducing the burden of IMD is to control serogroup B disease.

\section{Acknowledgments}

The authors acknowledge the role of staff in hospitals, laboratories and Public Health Units in collecting and reporting data on IMD cases in NSW.

This work was completed while Erin Passmore was a trainee on the NSW Public Health Officer Training Program, funded by the NSW Ministry of Health. She undertook this work while based at the Communicable Diseases Branch, Health Protection NSW.

\section{References}

1. Cohen NJ. Introduction of the National Meningococcal C Vaccination Program. Commun Dis Intell 2003; 27(2): 161-2.

2. Hull B, Dey A, Mahajan D, Campbell-Lloyd S, Menzies RI, McIntyre PB. NSW Annual Immunisation Coverage Report, 2009. N S W Public Health Bull 2010; 21(10): 210-23. doi:10.1071/NB10045

3. National Health and Medical Research Council. The Australian Immunisation Handbook. 10th ed. Canberra: Australian Government Department of Health and Ageing; 2013.
4. de Voer RM, van der Klis FR, Engels CW, Schepp RM, van de Kassteele J, Sanders EA et al. Kinetics of antibody responses after primary immunization with meningococcal serogroup $\mathrm{C}$ conjugate vaccine or secondary immunization with either conjugate or polysaccharide vaccine in adults. Vaccine 2009; 27(50): 6974-82. doi:10.1016/j.vaccine.2009.09.082

5. McAnulty J, Habib M, Muscatello D. Meningococcal disease in New South Wales. Commun Dis Intell 1997; 21(21): 322.

6. Hogan D, McAnulty J. Meningococcal disease in New South Wales, 1991-2002. N S W Public Health Bull 2004; 15(3): 39-43.

7. Chiu C, Dey A, Wang H, Menzies R, Deeks S, Mahajan D. Vaccine preventable diseases in Australia, 2005 to 2007. Commun Dis Intell 2010; 34(Suppl): S1-167.

8. Lahra M, Enriquez R. Annual report of the Australian Meningococcal Surveillance Program. Commun Dis Intell 2012; 36(3): E251-62.

9. NNDSS Annual Report Writing Group. Australia's notifiable diseases status, 2010: Annual report of the National Notifiable Diseases Surveillance System - Results: Other bacterial infections. Commun Dis Intell 2012; 36(1): 1-69.

10. Australian Government Department of Health and Ageing. National Notifiable Diseases Surveillance System. Disease notification rates, Australia, 1991-2012. Available at: http:// www9.health.gov.au/cda/source/rpt_2.cfm?RequestTime out=500 (Cited 13 November 2013).

11. Siu T, Tang W, Dawar M, Patrick DM. Impact of routine immunization using meningococcal $\mathrm{C}$ conjugate vaccine on invasive meningococcal disease in British Columbia. Can J Public Health 2008; 99(5): 380-2.

12. Balmer P, Borrow R, Miller E. Impact of meningococcal C conjugate vaccine in the UK. J Med Microbiol 2002; 51(9): $717-22$.

13. Larrauri A, Cano R, Garcia M. Mateo Sd. Impact and effectiveness of meningococcal $\mathrm{C}$ conjugate vaccine following its introduction in Spain. Vaccine 2005; 23(32): 4097-100. doi:10.1016/j.vaccine.2005.03.045

14. McIntyre PB, O’Brien KL, Greenwood B, van de Beek D. Effect of vaccines on bacterial meningitis worldwide. Lancet 2012; 380(9854): 1703-11. doi:10.1016/S0140-6736(12)61187-8

15. Trotter CL, Ramsay ME, Gray S, Fox A, Kaczmarski E. No evidence for capsule replacement following mass immunisation with meningococcal serogroup C conjugate vaccines in England and Wales. Lancet Infect Dis 2006; 6(10): 616-7. doi:10.1016/ S1473-3099(06)70584-9

16. Granoff DM. Review of meningococcal group B vaccines. Clin Infect Dis 2010; 50(Suppl 2): S54-65. doi:10.1086/648966

17. Viner RM, Booy R, Johnson H, Edmunds WJ, Hudson L, Bedford $\mathrm{H}$ et al. Outcomes of invasive meningococcal serogroup B disease in children and adolescents (MOSAIC): a case-control study. Lancet Neurol 2012; 11(9): 774-83. doi:10.1016/S14744422(12)70180-1

18. Borg J, Christie D, Coen PG, Booy R, Viner RM. Outcomes of meningococcal disease in adolescence: prospective, matchedcohort study. Pediatrics 2009; 123(3): e502-9. doi:10.1542/ peds.2008-0581

19. Guimont C, Hullick C, Durrheim D, Ryan N, Ferguson J, Massey P. Invasive meningococcal disease: improving management through structured review of cases in the Hunter New England area. Aust J Public Health 2010; 32(1): 38-43. 